https://doi.org/10.15407/ujpe64.7.566

F. FIONDA

on behalf of the ALICE Collaboration

Department of Physics and Technology, University of Bergen

(55, Allegaten, Bergen 500\%, Norway; e-mail: fiorella.fionda@cern.ch)

\title{
QUARKONIUM PRODUCTION MEASUREMENTS WITH THE ALICE DETECTOR AT THE LHC
}

\begin{abstract}
In (ultra-)relativistic heavy-ion collisions, the strongly interacting matter is predicted to undergo a phase transition into a plasma of deconfined quarks and gluons (QGP), and quarkonia probe different aspects of this medium. However, the medium modification of the quarkonium production includes also the contribution of cold nuclear matter effects (CNM), such as the shadowing or nuclear break-up in addition to QGP effects. Proton-nucleus collisions, where no $Q G P$ is expected, are used to measure cold nuclear matter effects on the quarkonium production. The vacuum production of quarkonia is modeled in proton-proton ( $p p$ ) collisions, which are used as the reference for both heavy-ion and proton-nucleus collisions. Besides serving as a reference, the results in pp collisions represent a benchmark test of QCD-based models in both perturbative and non-perturbative regimes. The ALICE detector has unique capabilities at the LHC for measuring quarkonia down to the zero transverse momentum. Measurements are carried out at both central and forward rapidities in the dielectron and dimuon decay channels, respectively. In this contribution, the latest quarkonium measurements performed by the ALICE Collaboration during the LHC Run-2 period for various energies and colliding systems will be discussed.
\end{abstract}

Ke ywords: QGP, quarkonium,relativistic heavy-ion collisions, cold nuclear matter effects.

\section{Physics Motivations}

Quarkonium measurements represent an important tool for the investigation of the interaction of heavy quarks with the hot and energy-dense medium created in heavy-ion collisions, known as Quark-Gluon Plasma (QGP) [1], and provide an important insight about its properties. In the original prediction by Matsui and Satz [2], it was argued that quarkonium states could melt in a deconfined medium, since the binding energy between the quark and antiquark is screened due to the presence of free color charges. This implies that the quarkonium production in heavy-ion collisions should be suppressed as compared to binary-scaled $p p$ collisions. However, it is also argued that the large production cross-section of heavy quarks in the hot thermalized medium leads to the (re)generation of quarkonia via the statistical recombination at the phase boundary [3] or through the coalescence of charm quarks [4]. Models including

(C) F. FIONDA, 2019 (re)generation describe the majority of charmonium measurements from LHC Run-1 (2009-2013), showing already the evidence that the (re)generation is the dominant production mechanism of $J / \psi$ in heavy-ion collisions at LHC energies [5]. Measurements of the bottomonium production, for which the contribution from the (re)generation could be small due to the much smaller beauty production cross-section, and the comparison with the corresponding charmonium results can further shed light on the quarkonium production mechanisms in large systems. Furthermore, if heavy-flavor quarks thermalize in the QGP, regenerated quarkonium states could inherit their flow and then participate in the collective motion of the QGP.

The study of the quarkonium production in proton-nucleus collisions is relevant to quantify cold nuclear matter (CNM) effects. Mechanisms such as a modification of the parton distribution functions in nuclei, the presence of a Color Glass Condensate (CGC), and coherent energy loss of the $c \bar{c}$ or $b \bar{b}$ pair in the medium have been employed to describe the

ISSN 2071-0194. Ukr. J. Phys. 2019. Vol. 64, No. 7 
$J / \psi$ and $\Upsilon$ production obtained in proton-nucleus collisions from the LHC Run-1 [6-8].

In elementary $p p$ collisions, the production of a quarkonium state can be understood as the creation of a heavy-quark pair $(q \bar{q})$ followed by its binding into a state with given quantum numbers. The first step is well described by perturbative quantum chromodynamics (QCD), while the second step is inherently non-perturbative. Currently, none of the existing models is able to satisfactorily describe simultaneously all aspects of the quarkonium production in $p p$ collisions. Therefore, more differential measurements represent a powerful tool for adding further constraints to quarkonium production models, improving significantly our understanding of quarkonium production mechanisms in elementary hadronic collisions.

\section{Quarkonium Measurements in ALICE}

The ALICE detector [9] has unique capabilities to measure the quarkonium production down to the zero transverse momentum $\left(p_{\mathrm{T}}\right)$ in two rapidity ranges ${ }^{1}$ : at mid-rapidity $(|y|<0.9)$ with the central barrel through the dielectron decay channel and at forward rapidity $(2.5<y<4)$ with the muon arm through the dimuon decay channel.

The main tracking detectors in the central barrel are the Inner Tracking System (ITS) and the Time Projection Chamber (TPC). The ITS provides the primary and secondary vertex information, the latter is useful to separate the non-prompt $J / \psi$ contribution (from beauty-hadron decays). The TPC provides the excellent particle identification for particles with intermediate momenta, in particular, for electrons up to about $10 \mathrm{GeV} / c$, based on the measurement of their specific energy loss.

The forward muon spectrometer includes a dipole magnet with an integrated field of $3 \mathrm{~T} \cdot \mathrm{m}$, five tracking stations comprising two planes of cathode pad chambers each, and two trigger stations consisting of two planes of resistive plate chambers each. The latter allows one to trigger on events with at least a pair of opposite-sign track segments in the muon trigger

\footnotetext{
1 The rapidity ranges are quoted in the "laboratory" reference frame $\left(y=y_{\text {lab }}\right)$ which is coincident with the center-of-mass reference frame $\left(y_{\mathrm{cms}}\right)$ in $p p$ and $\mathrm{Pb}-\mathrm{Pb}$ collisions, but not in $p$ - $\mathrm{Pb}$ collisions because of the asymmetric beam conditions.
}

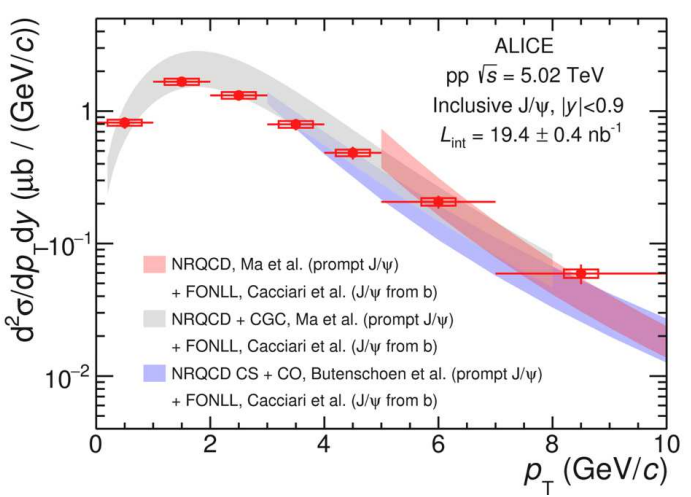

Fig. 1. $p_{\mathrm{T}}$-differential inclusive $J / \psi$ cross-section measured at mid-rapidity in $p p$ collisions at $\sqrt{s}=5 \mathrm{TeV}$ compared to prompt $J / \psi$ NRQCD calculations added to predictions of nonprompt $J / \psi$ from FONLL (see [10] and references therein)

system, each with a $p_{\mathrm{T}}$ above a specific threshold. A system of absorbers is used for filtering out hadrons.

\section{Results: Selected Highlights}

\section{1. pp collisions}

An extensive study of quarkonium production crosssections in $p p$ collisions has been performed by the ALICE Collaboration at several center-of-mass energies.

In Fig. 1, the inclusive $J / \psi$ cross-section measured at mid-rapidity at $\sqrt{s}=5 \mathrm{TeV}$ (see [10] and references therein) is compared to different sets of Non-Relativistic QCD (NRQCD) calculations of the prompt $J / \psi$ production.

The model from Ma et al. is coupled to a CGC description of the low- $x$ gluons in the proton and can predict the prompt $J / \psi$ cross-sections down to $p_{\mathrm{T}}=$ $=0$. In all cases, the non-prompt $J / \psi$ component calculated from Fixed-Order Next-To-Leading-Logarithm (FONLL) predictions is added to the prompt $J / \psi$ contribution.

The agreement between all models and data is good in the measured $p_{\mathrm{T}}$ range. It is worth noting that the uncertainties on the data points are significantly smaller than the model uncertainties, especially at low $p_{\mathrm{T}}$. The $\psi(2 \mathrm{~S})$-to- $J / \psi$ cross-section ratio, measured at the forward rapidity as a function of $p_{\mathrm{T}}$ in $p p$ collisions at $\sqrt{s}=13 \mathrm{TeV}$, is compared to NLO NRQCD calculations in Fig. 2 (see [11] and references therein). In the ratio, many of the systematic uncertainties cancel for both data and model. 


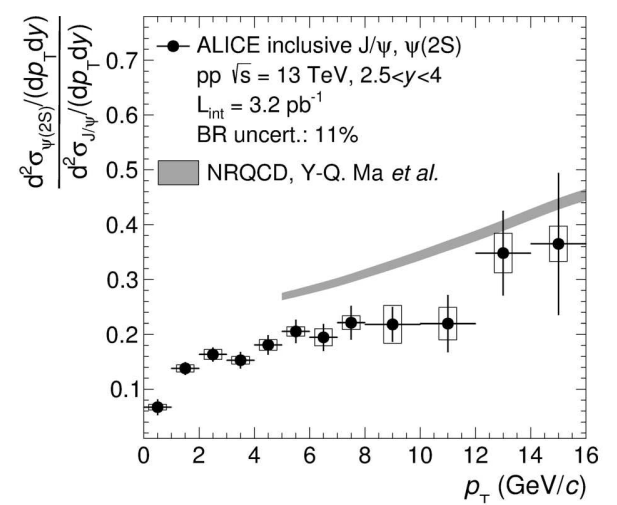

Fig. 2. $\psi(2 \mathrm{~S})$-to- $J / \psi$ cross-section ratio as a function of $p_{\mathrm{T}}$ in $p p$ collisions at $\sqrt{s}=13 \mathrm{TeV}$ measured at the forward rapidity as compared to NLO NRQCD calculations (see [11] and references therein)

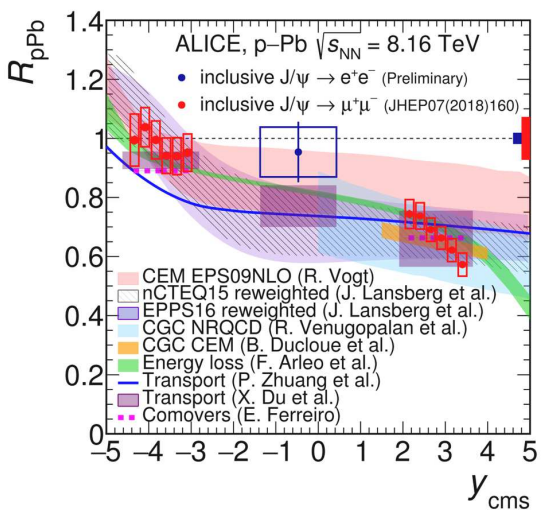

Fig. 3. $R_{p-\mathrm{Pb}}$ as a function of $y_{\mathrm{cms}}$ of the $J / \psi$ in $p$ - $\mathrm{Pb}$ collisions at $\sqrt{s}_{\mathrm{NN}}=8.16 \mathrm{TeV}$. The results are compared to several theoretical predictions (see [13] and references therein; for the model of $\mathrm{Du}$ et al. see [14])

From the comparison, it is clear that there are still tensions between data and models. Similarly, discrepancies are observed for polarization measurements performed in $p p$ collisions at $\sqrt{s}=8 \mathrm{TeV}$ at the forward rapidity [12].

\section{2. $\boldsymbol{p}-\mathrm{Pb}$ collisions}

The nuclear effects on the quarkonium production in $p$ - $\mathrm{Pb}$ collisions are estimated via the $p_{\mathrm{T}}$ and rapidity differential nuclear modification factor defined as

$R_{p-\mathrm{Pb}}\left(y_{\mathrm{cms}}, p_{\mathrm{T}}\right)=\frac{\mathrm{d}^{2} \sigma_{p-\mathrm{Pb}}^{\text {onium }} / \mathrm{d} y_{\mathrm{cms}} \mathrm{d} p_{\mathrm{T}}}{A_{\mathrm{Pb}} \mathrm{d}^{2} \sigma_{p p}^{\text {onium }} / \mathrm{d} y_{\mathrm{cms}} \mathrm{d} p_{\mathrm{T}}}$,

where the $p-\mathrm{Pb}$ production cross-section of a given quarkonium state, $\mathrm{d}^{2} \sigma_{p-\mathrm{Pb}}^{\text {onium }} / \mathrm{d} y_{\mathrm{cms}} \mathrm{d} p_{\mathrm{T}}$, is normalized to the corresponding quantity for $p p$ collisions times the atomic mass number of a $\mathrm{Pb}$ nucleus $\left(A_{\mathrm{Pb}}=\right.$ $=208)$. The $p_{\mathrm{T}}$-integrated $R_{p \text {-Pb }}$ of inclusive $J / \psi$, measured in $p$ - $\mathrm{Pb}$ collisions at $\sqrt{s_{\mathrm{NN}}}=8.16 \mathrm{TeV}$, is shown in Fig. 3 as a function of the center-of-mass rapidity, $y_{\mathrm{cms}}$. Measurements in the dimuon channel are performed by taking data in two configurations of the beams with either protons or $\mathrm{Pb}$ ions going toward the muon spectrometer, corresponding to forward and backward rapidities, respectively. For the mid-rapidity measurement, the data corresponding to the two configurations can be combined due to the symmetry of the central barrel detector. The nuclear modification factor is compatible with unity at backward and mid-rapidities. In contrast, a suppression is visible at the forward rapidity. It is compared to several theoretical models which attempt to describe the prompt $J / \psi$ production (see [13] and references therein; for the model of Du et al. see [14]). The results of calculations based on shadowing only show a good agreement with data, when the ${ }^{\text {CTEQ15 }}$ or EPPS16 set of nuclear parton distribution functions (nPDF) are adopted (Lansberg et al.), while using the EPS09 set of nPDF leads to a slightly worse agreement at the forward $y_{\mathrm{cms}}$ (Vogt). Calculations based on a CGC approach coupled with various quarkonium vacuum production models are able to reproduce the data in their domain of validity, corresponding to the forward- $y_{\mathrm{cms}}$ region (Venugopalan et al.; Ducloue et al.). The model of Arleo et al., based on the calculation of the effects of parton coherent energy loss, gives a good description of the results for both backward$y_{\mathrm{cms}}$ and forward- $y_{\mathrm{cms}}$ rapidities. Finally, models including a contribution from the final state interactions of the $c \bar{c}$ pair with the partonic/hadronic system created in the collision (Zhuang et al.; Du et al.; Ferreiro) can also reproduce the trend observed in the data. In the latter set of models, the nuclear shadowing is included, and it is the mechanism that plays a dominant role in determining the values of the nuclear modification factors.

The $R_{p-\mathrm{Pb}}$ for $\psi(2 \mathrm{~S})$ as a function of $y_{\mathrm{cms}}$ is shown in Fig. 4, where it is compared to the corresponding $\mathrm{J} / \psi$ result. At the forward rapidity, $J / \psi$ and $\psi(2 \mathrm{~S})$ show a similar suppression, while, at the backward rapidity, $\psi(2 \mathrm{~S})$ is significantly more suppressed than $J / \psi$. Contrary to the $J / \psi$ case, only models that include final state interactions with the surrounding medium are able to reproduce $\psi(2 \mathrm{~S})$ results.

ISSN 2071-0194. Ukr. J. Phys. 2019. Vol. 64, No. 7 
The ALICE Collaboration has also measured long-range correlations between forward- $y_{\mathrm{cms}}$ and backward- $y_{\mathrm{cms}}$ inclusive $J / \psi$ and mid-rapidity charged hadrons, in $p$ - $\mathrm{Pb}$ collisions at both $\sqrt{s}_{\mathrm{NN}}=$ $=5.02$ and $8.16 \mathrm{TeV}$ [15]. The data indicate persisting long-range correlation structures at $\Delta \phi \sim 0$ and $\Delta \phi \sim \pi$, reminiscent of the double ridge previously found in charged-particle correlations at midand forward rapidities [16]. The corresponding $v_{2}^{J / \psi}$, obtained by combining data of the two collision energies, is shown in Fig. 5. In heavy-ion collisions, this coefficient is related to the azimuthal anisotropy of the final-state particle momentum distribution and is sensitive to the geometry and the dynamics of the early stages of the collision. The results in $p-\mathrm{Pb}$ collisions are compared to $v_{2}^{J / \psi}$ measurements performed in $\mathrm{Pb}-\mathrm{Pb}$ collisions at $\sqrt{s_{\mathrm{NN}}}=5.02 \mathrm{TeV}$ [17]. The positive $v_{2}$ coefficients observed in $\mathrm{Pb}-\mathrm{Pb}$ collisions for $p_{\mathrm{T}}^{J / \psi}$ below $3-4 \mathrm{GeV} / c$ are believed to originate from the recombination of charm quarks thermalized in the medium and are described fairly well by the transport model. In $p$ - $\mathrm{Pb}$ collisions, the $v_{2}^{J / \psi}$ is compatible with zero at low $p_{\mathrm{T}}$, and this is in line with expectations, since no QGP is expected to be produced in which charm quarks could thermalize. Even assuming such scenario, the amount of produced charm quarks is small compared to that in heavy-ion collisions. Therefore, the contribution from the recombination should be negligible. However, at high- $p_{\mathrm{T}}$, $J / \psi v_{2}$ is comparable to the magnitude of the flow observed in central $\mathrm{Pb}-\mathrm{Pb}$ collisions. It is worth noting that, in $\mathrm{Pb}-\mathrm{Pb}$ collisions, the measured $v_{2}^{J / \psi}$ coefficients exceed substantially the theoretical predictions for $p_{\mathrm{T}}^{J / \psi}>4 \mathrm{GeV} / c$, where the main contribution to $v_{2}^{J / \psi}$ is expected to come from the path-length dependent suppression inside the medium. These intriguing results point to a common underlying mechanism, not included in current calculations, at the origin of the comparable magnitude of the $v_{2}^{J / \psi}$ at a high transverse momentum in both systems.

\section{3. $\mathrm{Pb}-\mathrm{Pb}$ collisions}

The nuclear modification factor, for a quarkonium state in a given centrality class $i$ of the $\mathrm{Pb}-\mathrm{Pb}$ collision, is calculated as

$R_{\mathrm{Pb}-\mathrm{Pb}}^{1}\left(y, p_{\mathrm{T}}\right)=\frac{\mathrm{d}^{2} N_{\mathrm{Pb}-\mathrm{Pb}, i}^{\text {onium }} / \mathrm{d} y \mathrm{~d} p_{\mathrm{T}}}{\left\langle T_{\mathrm{AA}}^{i}\right\rangle \mathrm{d}^{2} \sigma_{p p}^{\text {onium }} / \mathrm{d} y \mathrm{~d} p_{\mathrm{T}}}$,

ISSN 2071-0194. Ukr. J. Phys. 2019. Vol. 64, No. 7

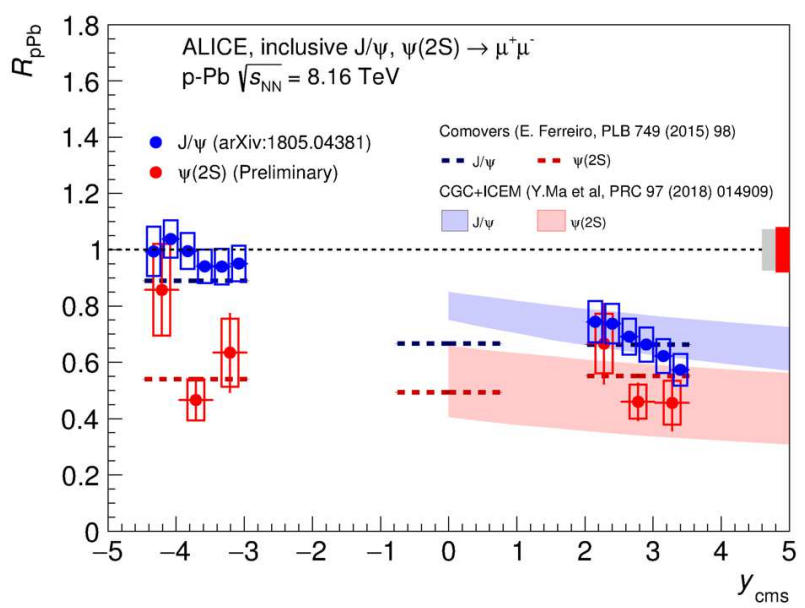

Fig. 4. $R_{p-\mathrm{Pb}}$ as a function of $y_{\mathrm{cms}}$ for $\psi(2 \mathrm{~S})$ and $J / \psi$ in $p-\mathrm{Pb}$ collisions at $\sqrt{s}_{\mathrm{NN}}=8.16 \mathrm{TeV}$. The results are compared to different theoretical models (see references on the plot)

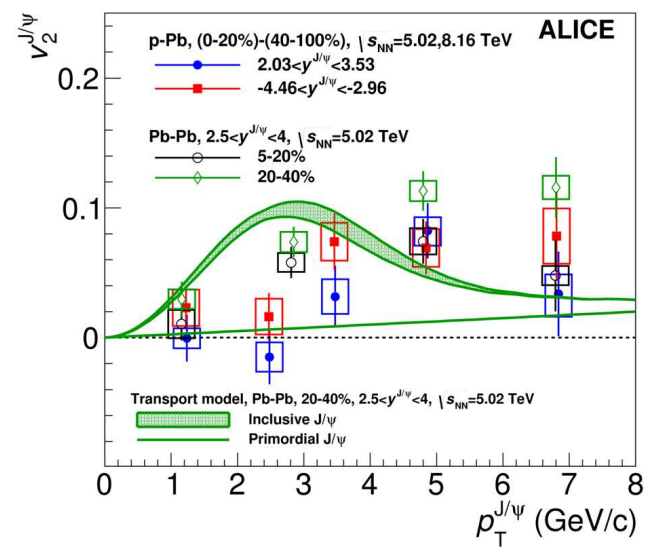

Fig. 5. Combined $v_{2}^{J / \psi}$ coefficients in $p-\mathrm{Pb}$ collisions at $\sqrt{s_{\mathrm{NN}}}=5.02$ and $8.16 \mathrm{TeV}$ compared to results in central and semicentral $\mathrm{Pb}-\mathrm{Pb}$ collisions at $\sqrt{s_{\mathrm{NN}}}=5.02 \mathrm{TeV}$ and to the transport model calculations for semicentral $\mathrm{Pb}-\mathrm{Pb}$ collisions (see [15] and references therein).

where $\mathrm{d}^{2} N_{\mathrm{Pb}-\mathrm{Pb}, i}^{\mathrm{Onium}} / \mathrm{d} y \mathrm{~d} p_{\mathrm{T}}$ is the corrected yield of the studied quarkonium state in $\mathrm{Pb}-\mathrm{Pb}$ collisions, $\left\langle T_{\mathrm{AA}}^{i}\right\rangle$ is the nuclear overlap function, and $\mathrm{d}^{2} \sigma_{\mathrm{pp}}^{\text {onium }} / \mathrm{d} y \mathrm{~d} p_{\mathrm{T}}$ is the corresponding cross-section in $p p$ collisions at the same center-of-mass energy. Figure 6 shows $R_{\mathrm{AA}}$ as a function of the centrality, for $J / \psi$ measured at the forward rapidity in $\mathrm{Pb}-\mathrm{Pb}$ collisions at $\sqrt{s_{\mathrm{NN}}}=5.02 \mathrm{TeV}$, in the transverse momentum range $0.3<p_{\mathrm{T}}<<8 \mathrm{GeV} / c[18]$. The $p_{\mathrm{T}}$ region below $0.3 \mathrm{GeV} / c$ was excluded in order to reduce significantly the contribution from the photo-production 


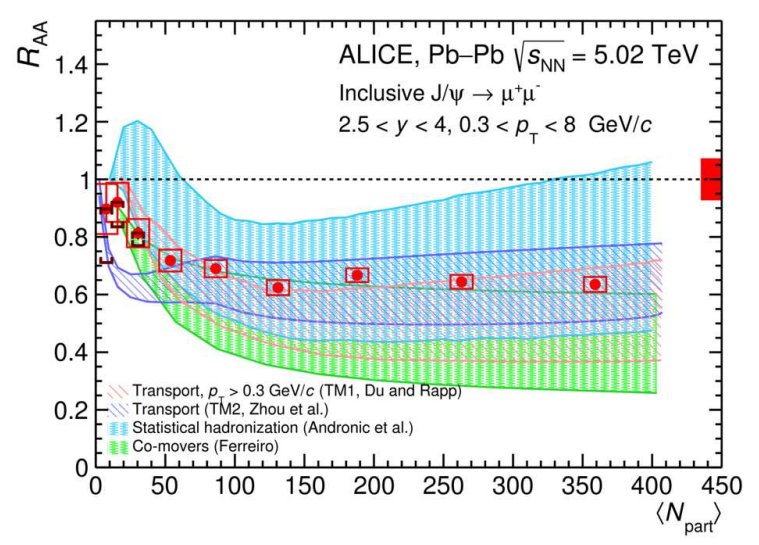

Fig. 6. Centrality dependence of inclusive $\mathrm{J} / \psi R_{\mathrm{AA}}$ for $0.3<$ $<p_{\mathrm{T}}<8 \mathrm{GeV} / c$ measured in $\mathrm{Pb}-\mathrm{Pb}$ collisions at $\sqrt{s_{\mathrm{NN}}}=$ $=5.02 \mathrm{TeV}$ and comparison with theoretical models (see [18] and references therein)

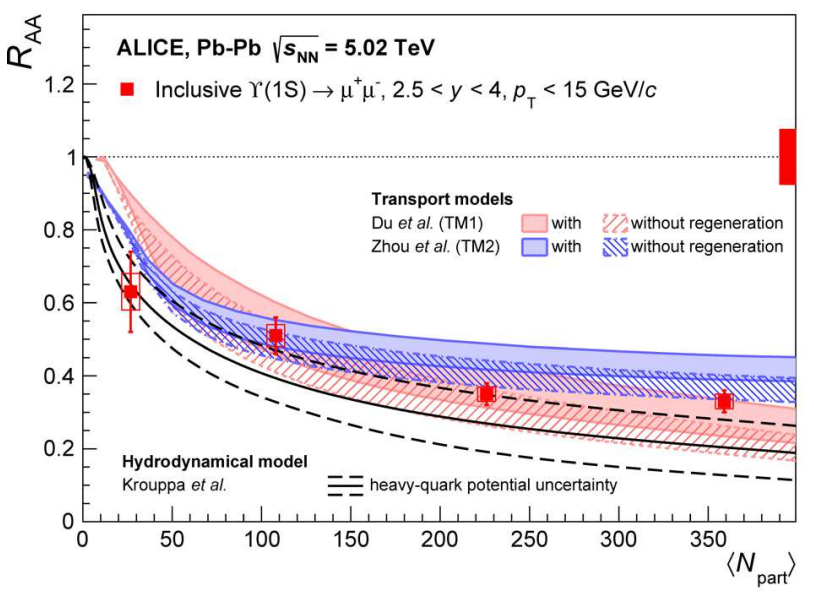

Fig. 7. Inclusive $\Upsilon(1 \mathrm{~S}) R_{\mathrm{AA}}$ as a function of the centrality measured at the forward rapidity in $\mathrm{Pb}-\mathrm{Pb}$ collisions at $\sqrt{s_{\mathrm{NN}}}=5.02 \mathrm{TeV}$, compared to theoretical model calculations (see [20] and references therein)

of $J / \psi$, which could influence the $R_{\mathrm{AA}}$ in peripheral collisions [19]. The results are compared to several theoretical models. The statistical hadronization model assumes that $J / \psi$ are created, like all other hadrons, only at the chemical freeze-out according to their statistical weights. Transport models are based on a thermal rate equation, which includes the continuous dissociation and regeneration of $J / \psi$, both in the QGP and in the hadronic phase. Finally, in the "co-mover" model, $J / \psi$ are dissociated via interactions with the partons/hadrons produced in the same rapidity range, and the regeneration term is included

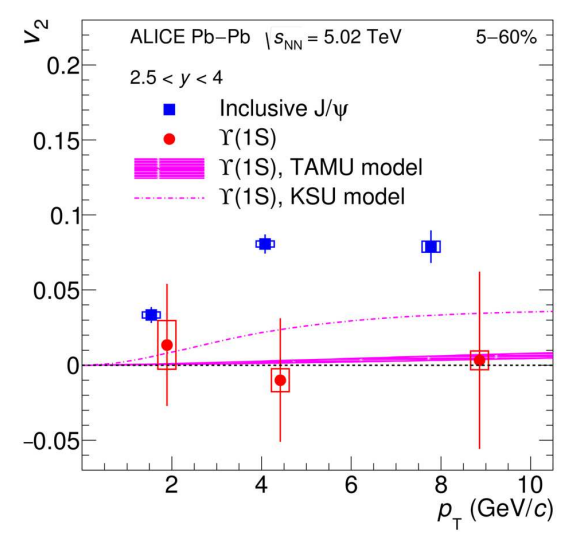

Fig. 8. The $\Upsilon(1 S) v_{2}$ coefficient as a function of $p_{\mathrm{T}}$ measured in $\mathrm{Pb}-\mathrm{Pb}$ collisions at $\sqrt{s_{\mathrm{NN}}}=5.02 \mathrm{TeV}$ in the $5-60 \%$ centrality interval compared to that of inclusive $J / \psi$ and to theoretical calculations (see [21] and references therein)

as well. The data are described by the various calculations, the latter having rather large uncertainties. These are related to the choice of the corresponding input parameters, and in particular, the nucleonnucleon c $\overline{\mathrm{c}}$ production cross-section $\left(\mathrm{d} \sigma_{\mathrm{c} \overline{\mathrm{c}}} / \mathrm{d} y\right)$, as well as the set of nPDF.

The centrality dependence of the nuclear modification factor for $\Upsilon(1 \mathrm{~S})$ measured in $\mathrm{Pb}-\mathrm{Pb}$ collisions at $\sqrt{s_{\mathrm{NN}}}=5.02 \mathrm{TeV}$ is shown in Fig. 7 along with several theoretical model calculations [20]. Both transport and dynamical model calculations reproduce qualitatively the observed centrality dependence. However, current uncertainties on both model and data prevent a firm conclusion regarding the contribution from the regeneration in the bottomonium sector. Furthermore, more precise measurements of the feed-down contribution from higher-mass bottomonia to the $\Upsilon(1 S)$ are needed for a correct interpretation of results. Further information about the interplay between the regeneration and suppression in the bottomonium sector can be provided by elliptic flow measurements. The $v_{2}$ of $\Upsilon(1 \mathrm{~S})$, obtained by combining data samples recorded by ALICE during the 2015 and $2018 \mathrm{LHC} \mathrm{Pb-Pb}$ runs at $\sqrt{s_{\mathrm{NN}}}=5.02 \mathrm{TeV}$, is shown in Fig. 8 in three $p_{\mathrm{T}}$ intervals [21] and is compared to the inclusive $J / \psi v_{2}$, measured in the same centrality and rapidity ranges. The $\Upsilon(1 \mathrm{~S})$ results are compatible with zero and with the small positive values predicted by the available theoretical models within uncertainties. Furthermore, the $\Upsilon(1 \mathrm{~S})$ $v_{2}$ is found to be lower by about $2.6 \sigma$ compared to the

ISSN 2071-0194. Ukr. J. Phys. 2019. Vol. 64, No. 7 
one of the inclusive $J / \psi$ in the centrality $5-60 \%$ and for $2<p_{\mathrm{T}}<15 \mathrm{GeV} / c$. This observation, coupled to the different measured centrality and $p_{\mathrm{T}}$ dependences of the $\Upsilon(1 \mathrm{~S})$ and $J / \psi$ suppression, provides a further evidence that, unlike $\Upsilon(1 \mathrm{~S})$, the $J / \psi$ production has a significant regeneration component.

\section{Conclusions and Future Perspectives}

Selected quarkonium measurements in $p p, p-\mathrm{Pb}$, and $\mathrm{Pb}-\mathrm{Pb}$ collisions performed by the ALICE Collaboration are presented. In $p p$ collisions, NRQCD predictions coupled with CGC fairly describe the data in a wide range of momentum and rapidity. However, some tensions between data and models are still present. In $p$ - $\mathrm{Pb}$ collisions, theoretical models are in fair agreement with quarkonium results, in particular, for $\psi(2 \mathrm{~S})$, models that include final state effects are able to describe the data. The positive $v_{2}$ measured for $J / \psi$ is comparable with a similar measurement in $\mathrm{Pb}-\mathrm{Pb}$ collisions for $p_{\mathrm{T}}>44 \mathrm{GeV} / c$. The latter exceeds theoretical predictions in $\mathrm{Pb}-\mathrm{Pb}$ collisions at high $p_{\mathrm{T}}$, where the $v_{2}$ originates from the path-length suppression inside the medium. This intriguing observation points to a common mechanism at the origin of $v_{2}$ in both systems at high transverse momentum, besides what is currently included in the models. An extensive $y$ and $p_{\mathrm{T}}$-differential studies of the $J / \psi$ suppression in $\mathrm{Pb}-\mathrm{Pb}$ collisions indicate that, at $\mathrm{LHC}$ energies, a significant contribution to the $J / \psi$ yields originates from the regeneration mechanism. However, for a better discrimination among the models, an improved precision is needed for both data and theoretical predictions. $\Upsilon(1 S)$ is found to be more suppressed than $J / \psi$. Currently, the comparison with models does not allow us to quantify the contribution from the regeneration. A large elliptic flow for $J / \psi$, measured at low $p_{\mathrm{T}}$, suggests the thermalization of charm quarks within the medium. On the contrary, the $\Upsilon(1 \mathrm{~S}) v_{2}$ is found to be compatible with zero and with values predicted by models, suggesting a negligible contribution from the regeneration mechanism in the bottonomium sector.

A significant improvement regarding the quarkonium measurements is expected for Run-3 (starting in 2021) and Run-4, when a major upgrade of the ALICE detector is foreseen [22]. A high-statistics minimum bias sample $\left(L_{\text {int }}=10 \mathrm{nb}^{-1}\right)$ will improve significantly mid-rapidity quarkonium measurements at low transverse momenta. Furthermore, a new Muon Forward Tracker (MFT) will be installed at the forward rapidity enabling the reconstruction of secondary vertices in this rapidity range, needed to measure the contribution of charmonia coming from beauty-hadron decays.

1. N. Cabibbo, G. Parisi. Exponential hadronic spectrum and quark liberation. Phys. Lett. B 59, 67 (1975).

2. T. Matsui, H. Satz. $J / \psi$ suppression by quark-gluon plasma formation. Phys. Lett. B 178, 416 (1986).

3. P. Braun-Munzinger, J. Stachel. (Non)thermal aspects of charmonium production and a new look at $J / \psi$ suppression. Phys. Lett. B 490, 196 (2000).

4. R.L. Thews, M. Schroedter, J. Rafelski. Enhanced $J / \psi$ production in deconfined quark matter. Phys. Rev. C 63, 054905 (2001).

5. B. Abelev et al., ALICE Coll. $J / \psi$ Suppression at Forward Rapidity in $\mathrm{Pb}-\mathrm{Pb}$ Collisions at $\sqrt{s_{\mathrm{NN}}}=2.76 \mathrm{TeV}$. Phys. Rev. Lett. 109, 072301 (2012).

6. B. Abelev et al., ALICE Coll. $J \psi$ production and nuclear effects in $p-\mathrm{Pb}$ collisions at $\sqrt{s_{\mathrm{NN}}}=5.02 \mathrm{TeV}$. JHEP 02, 73 (2014).

7. J. Adam et al., ALICE Coll. Centrality dependence of inclusive $J \psi$ production in $p-\mathrm{Pb}$ collisions at $\sqrt{s_{\mathrm{NN}}}=$ $=5.02 \mathrm{TeV}$. JHEP 11, 127 (2015).

8. B. Abelev et al., ALICE Coll. Production of inclusive $\Upsilon(1 \mathrm{~S})$ and $\Upsilon(2 \mathrm{~S})$ in $p$-Pb collisions at $\sqrt{s_{\mathrm{NN}}}=5.02 \mathrm{TeV}$. Phys. Lett. B 740, 105 (2015).

9. K. Aamodt et al., ALICE Coll. The ALICE experiment at the CERN LHC. JINST 3, S08002 (2008).

10. S. Acharya et al., ALICE Coll. Inclusive $J / \psi$ production at mid-rapidity in $p p$ collisions at $\sqrt{s}=5.02 \mathrm{TeV}$. arXiv:1905.07211 [nucl-ex] (2019).

11. S. Acharya et al., ALICE Coll. Energy dependence of forward-rapidity $J / \psi$ and $\psi(2 \mathrm{~S})$ production in $p p$ collisions at the LHC. Eur. Phys. J. C 77, 392 (2017).

12. S. Acharya et al., ALICE Coll. Measurement of the inclusive $J / \psi$ polarization at forward rapidity in $p p$ collisions at $\sqrt{\mathbf{s}}=\mathbf{8} \mathrm{TeV}$. Eur. Phys. J. C 78, 562 (2018).

13. S. Acharya et al., ALICE Coll. Inclusive $J / \psi$ production at forward and backward rapidity in $p$-Pb collisions at $\sqrt{s_{\mathrm{NN}}}=8.16 \mathrm{TeV}$. JHEP 07, 160 (2018).

14. X. Du, R. Rapp. In-medium charmonium production in proton-nucleus collisions. JHEP 03, 15 (2019).

15. S. Acharya et al., ALICE Coll. Search for collectivity with azimuthal $J / \psi$-hadron correlations in high multiplicity $p$ $\mathrm{Pb}$ collisions at and $\sqrt{\mathbf{s}_{\mathrm{NN}}}=\mathbf{5 . 0 2}$ and $8.16 \mathrm{TeV}$. Phys. Lett. B 780, 7 (2018).

16. V. Khachatryan et al., CMS Coll. Observation of longrange near-side angular correlations in proton-lead collisions at the LHC. Phys. Lett. B 718, 795 (2013).

17. S. Acharya et al., ALICE Coll. $J / \psi$ elliptic flow in $\mathrm{Pb}-$ $\mathrm{Pb}$ collisions at $\sqrt{\mathbf{S}_{\mathrm{NN}}}=\mathbf{5 . 0 2} \mathrm{TeV}$. Phys. Rev. Lett. 119, 242301 (2017).

18. J. Adam et al., ALICE Coll. $J / \psi$ suppression at forward rapidity in $\mathrm{Pb}-\mathrm{Pb}$ collisions at $\sqrt{\mathbf{s}_{\mathrm{NN}}}=\mathbf{5 . 0 2} \mathrm{TeV}$. Phys. Lett. B 766, 212 (2017). 
19. J. Adam et al., ALICE Coll. Measurement of an excess in the yield of $J / \psi$ at very low $p_{T}$ in $\mathrm{Pb}-\mathrm{Pb}$ Collisions at $\sqrt{s_{\mathrm{NN}}}=2.76 \mathrm{TeV}$. Phys. Rev. Lett. 116, 222301 (2016).

20. S. Acharya et al., ALICE Coll. $\Upsilon(1 \mathrm{~S})$ suppression at forward rapidity in $\mathrm{Pb}-\mathrm{Pb}$ collisions at $\sqrt{\mathbf{s}_{\mathrm{NN}}}=\mathbf{5 . 0 2} \mathrm{TeV}$. Phys. Lett. B 790, 89 (2019).

21. S. Acharya et al., ALICE Coll. Measurement of $\Upsilon(1 \mathrm{~S})$ elliptic flow at forward rapidity in $\mathrm{Pb}-\mathrm{Pb}$ collisions at $\sqrt{\mathbf{s}_{\mathrm{NN}}}=\mathbf{5 . 0 2} \mathrm{TeV}$. arXiv:1907.03169 [nucl-ex] (2019).

22. Z. Citron et al. Future physics opportunities for highdensity QCD at the LHC with heavy-ion and proton beams. arXiv:1812.06772 [hep-ph] (2018).

Received 08.07.19

Ф. Фіонда, від імені Колабораціӥ ALICE

ВИМІРЮВАННЯ ПРОДУКУВАННЯ

КВАРКОНІЯ ЗА ДОПОМОГОЮ ДЕТЕКТОРА

ALICE HA LHC

$\mathrm{P}$ е $з$ ю м е

Передбачається, що в (ультра)релятивістських зіткненнях важких іонів сильно взаємодіюча речовина проходить фа- зовий перехід до плазми кварків та глюонів (КГП), а кварконій може бути джерелом інформації щодо властивостей цієї матерії. Проте модифікація середовища, де продукується кварконій, включає також вплив холодної ядерної речовини (CNM) як екранування ядерного (брейкап) розвалу на додаток до ефектів КГП. Протон-ядерні зіткнення, в яких не очікуються утворення КГП, служать для визначення впливу холодної ядерної речовини на продукування кварконія. Вакуумне продукування кварконія моделюється в протон-протонних зіткненнях, які служать еталоном як для зіткнень важких іонів, так і для протон-ядерних зіткнень. Окрім калібровки, результати зіткнень протонів служать також орієнтиром для моделей, основаних на КХД як в пертурбативній, так і в непертурбативній областях. Детектор ALICE має унікальні для LHC можливості для вимірювання кварконіїв аж до нульового значення поперечного імпульсу. Вимірювання було виконано як для центральних, так і для передніх бистрот в каналах розпаду, відповідно, діелектрона та дімюона. В даній роботі представлено новітні вимірювання продукування кварконія Колаборацією ALICE на LHC під час Сеансу-2 при різних енергіях та для різних систем. 